Epidemiology and Infection

cambridge.org/hyg

\section{Original Paper}

Cite this article: Pebody RG, Boddington NL, Green HK, Bolotin S, Charlett A, Watson JM (2018). Assessing the severity of influenza: a role for longitudinal telephone surveys? Epidemiology and Infection 146, 2042-2048. https://doi.org/10.1017/S0950268818002261

Received: 5 February 2018

Revised: 7 July 2018

Accepted: 11 July 2018

First published online: 15 August 2018

\section{Key words:}

Epidemiology; pneumococci; vaccine policy development; vaccine preventable diseases; vaccines

\section{Author for correspondence:}

R. G. Pebody, E-mail: Richard.Pebody@phe. gov.uk

\title{
Assessing the severity of influenza: a role for longitudinal telephone surveys?
}

\author{
R. G. Pebody ${ }^{1}$, N. L. Boddington ${ }^{1}$, H. K. Green ${ }^{1}$, S. Bolotin ${ }^{1}$, A. Charlett ${ }^{1}$ \\ and J. M. Watson ${ }^{2}$
} ${ }^{1}$ National Infection Service, Public Health England, London, UK and ${ }^{2}$ Health Protection Directorate, Public Health
England, UK

\begin{abstract}
During the 2009 influenza pandemic, a rapid assessment of disease severity was a challenge as a significant proportion of cases did not seek medical care; care-seeking behaviour changed and the proportion asymptomatic was unknown. A random-digit-dialling telephone survey was undertaken during the 2011/12 winter season in England and Wales to address the feasibility of answering these questions. A proportional quota sampling strategy was employed based on gender, age group, geographical location, employment status and level of education. Households were recruited pre-season and re-contacted immediately following peak seasonal influenza activity. The pre-peak survey was undertaken in October 2011 with 1061 individuals recruited and the post-peak telephone survey in March 2012. Eight hundred and thirty-four of the 1061 (78.6\%) participants were successfully re-contacted. Their demographic characteristics compared well to national census data. In total, $8.4 \%$ of participants self-reported an influenza-like illness (ILI) in the previous 2 weeks, with $3.2 \%$ conforming to the World Health Organization (WHO) ILI case definition. In total, 29.6\% of the cases reported consulting their general practitioner. $54.1 \%$ of the 1061 participants agreed to be re-contacted about providing biological samples. A population-based cohort was successfully recruited and followed up. Longitudinal survey methodology provides a practical tool to assess disease severity during future pandemics.
\end{abstract}

\section{Introduction}

Rapid assessment of the severity of illness due to a new emerging pandemic influenza virus is recognised to be central to informing optimal prevention and control activities $[1,2]$. Severity, in this context, is taken to mean a function of both the infection severity of the average case (i.e. the likelihood that an infection is symptomatic; results in hospitalisation or death) and the infection attack rate in the general population.

During the 2009 influenza pandemic, surveillance in many countries relied on established clinical and virological systems based primarily on symptomatic patients in contact with health care systems through hospital or general practitioner (GP)-based surveillance systems for influenza-like illness (ILI) or acute respiratory illness. This risked overestimating severity, as 'milder' cases that were not in contact with health care services were missed [3, 4]. In England, this information was supplemented by a newly established treatment system, the National Pandemic Flu Service, used to administer health advice and antivirals to those presenting with clinical symptoms consistent with pandemic influenza [5].

Assessment of the true clinical attack rate in the community and determining infection severity in near real time proved to be challenging in 2009 through these traditional influenza surveillance systems [2]. Initial reports from Mexico were of cases of severe illness due to a swine-origin influenza virus, which raised international concern of a transmissible novel influenza virus with high infection severity $[6,7]$. Ultimately, it became apparent that, although this new pandemic virus was indeed transmissible from person-to-person, a large proportion of human infections was asymptomatic or resulted in only mild illness with cases not seeking medical care [8]. This clinical spectrum of illness led to an initial underestimation of the true total number of people infected and an overestimation of the infection severity. Furthermore, as public perception of the threat of the infection changed over the course of the pandemic, care-seeking behaviour also altered. These features led to difficulties in interpreting routine surveillance data [9]. The need for early population-based serological data during the pandemic was also identified as a key requirement $[2,10]$. Influenza serological surveys were felt necessary to provide early, accurate measures of susceptibility and recent infection. These can then be used to estimate the proportion of influenza infections that were asymptomatic, measure age-specific population susceptibility, estimate population infection attack rates and finally to contribute to measures of infection severity [10]. Following the 2009 pandemic, questions were raised and recommendations were made about the need during future 
pandemics of appropriate and timely serological data including representativeness of the convenience sampled surveys and the need for key epidemiological information on the samples, such as subject vaccination status [11].

Community-based surveillance can potentially address these various issues. The aim of such studies is to identify all infections, including those in people that do not access health care services. Linked to serological sampling, they can also potentially identify those that had an asymptomatic or only mildly symptomatic infection [12]. A number of pandemic community surveillance initiatives were undertaken in the UK in 2009, including internet-based surveys (FluSurvey) [13], serial residual serosurveys [14] and a household cohort survey (FluWatch) [15]. These approaches, however, raised issues of representativeness and cost. Longitudinal surveys by means of telephone have been identified as a potential alternative approach that could provide in near real time, population-based data on rates of ILI in the general population, an understanding of health care-seeking behaviour and vaccination status and how these measures change over time $[16,17]$. These surveys could also be used as a mechanism to undertake population-based biological sampling.

Following the evaluation of the 2009 UK pandemic surveillance response [11], a pilot Community Influenza Surveillance telephone survey was undertaken in England and Wales during the 2011/12 influenza season. This pilot survey aimed to collect self-reported data on the clinical and epidemiological features of seasonal influenza in the general population, together with information on health care-seeking behaviour and attitudes to such studies in order to evaluate the potential utility of this type of surveillance system for pandemic influenza. The key objectives were to estimate (by age, sex, geographical area and risk group status) the rate of recent self-reported clinical ILI, health care-seeking behaviour for ILI (such as GP visits), uptake of current and prior seasonal influenza vaccine and the acceptability of undertaking biological sampling.

\section{Methods}

Individuals were recruited using a population-based household telephone survey with proportional quota sampling by a market research company (Ipsos MORI). Information on each recruited individual and their household were collected both pre-season (October 2011) and immediately following the peak of seasonal influenza activity as defined by Public Health England (PHE) routine influenza surveillance data (March 2012). Most of the questions asked during the pre-peak survey were asked again post-peak for confirmation or status change of the interviewee. The variables addressing the key objectives such as ILI symptoms in the previous 2 weeks and questions about care-seeking behaviour were asked only during the post-peak survey.

\section{Study design}

The survey utilised a random-digit-dialling telephone sampling method, an established sampling approach to recruit a quota sample of households [18] by assigning a random telephone number to known, valid area codes in England [19]. The sampling frame included all households in England and Wales that have a landline telephone, which is currently estimated to be $88 \%$ [20]. To increase representativeness, quota sampling was employed to boost numbers from groups that are usually hard to reach using telephone surveys, such as males or younger age groups. Quotas were based on gender, age group, geographical region of residence, employment status and level of education attained. Quota sampling by employment status was utilised to avoid oversampling segments of the population who do not work full time.

\section{Data collection}

During the initial telephone call, participants provided background information about themselves and their household, as well as on any self-reported underlying clinical risk factors which may increase their likelihood of developing severe disease. Information was also collected on their influenza vaccination status for the 2011/12 seasonal influenza vaccine at the time of initial contact and vaccination status in 2010/11 and 2009/10. Participants were also asked about their willingness to be contacted by PHE to provide biological samples such as blood or selfsampling by finger prick and oral fluid for testing before and after the influenza season.

After the peak of influenza activity (March 2012), the same participants and their households were re-contacted and asked about any change in influenza vaccination status, whether they had experienced a self-defined ILI and any specific respiratory symptoms they had suffered in the previous 2 weeks as well as related health care-seeking behaviour they had undertaken, such as GP visits, visits to hospital or calls to telephone health advice service NHS Direct.

Influenza attack rates were calculated based on the selfreported ILI. The self-reported symptoms most closely matching the 2011 World Health Organization (WHO) definition for ILI of 'elevated temperature $\geqslant 38^{\circ} \mathrm{C}$ and cough with onset in the past seven days [21]' was used to create a modified WHO case definition of 'sudden onset of fever and cough in the previous two weeks'.

\section{Data analysis}

Demographic information was compared with various national data sources including national population data from the Office for National Statistics (ONS) [22] and the Labour Force Survey 2011 [23]. The population demographics used from ONS (2010) were for gender, age group and geographical region and the Labour Force Survey 2010 for education and employment. Self-reported influenza vaccination uptake and risk factor data were compared with national uptake data collected through ImmForm, a primary care-based influenza vaccine uptake monitoring surveillance system, based on extraction of electronic health records from GP information systems [24].

It is important to note that the quota sampling method used is not a probabilistic sample of the population, and thus it is not possible to formally estimate a variance, and thus confidence intervals around sample estimates.

\section{Survey size}

This was based on the available resource to recruit approximately 1000 individuals, rather than formal power calculations.

\section{Consent, ethics and confidentiality}

The National Research Ethics Service was approached regarding the need for ethical approval to carry out the survey. It was determined that the survey was considered a surveillance activity 
Table 1. Demographic characteristics of the individuals and households recruited in pre- and post-peak telephone survey with national data and the source, England and Wales, 2011-12

\begin{tabular}{|c|c|c|c|c|}
\hline & \multicolumn{2}{|c|}{ Survey $^{a}$} & & \\
\hline & \multirow{2}{*}{$\begin{array}{l}\text { Completed two surveys (\%) } \\
\qquad(n=834)\end{array}$} & \multirow{2}{*}{$\begin{array}{l}\text { Lost to follow-up (\%) } \\
\qquad(n=227)\end{array}$} & \multicolumn{2}{|c|}{ National } \\
\hline & & & National data (\%) & National source \\
\hline \multicolumn{5}{|l|}{ Gender } \\
\hline Male:female ratio & 0.8 & 0.9 & 1.0 & ONS 2010 \\
\hline \multicolumn{5}{|l|}{ Age group (\%) } \\
\hline $18-24$ years & 7.5 & 11.9 & 12.1 & \multirow[t]{4}{*}{ ONS 2010} \\
\hline 25-44 years & 28.8 & 35.2 & 34.6 & \\
\hline 45-64 years & 36.0 & 32.2 & 32.2 & \\
\hline $65+$ years & 27.7 & 20.7 & 21.0 & \\
\hline \multicolumn{5}{|l|}{ Region (\%) } \\
\hline NE & 6.0 & 6.2 & 4.7 & \multirow[t]{10}{*}{ ONS 2010} \\
\hline$Y \& H$ & 8.6 & 9.7 & 9.6 & \\
\hline EM & 9.1 & 5.3 & 8.1 & \\
\hline $\mathrm{EE}$ & 9.9 & 9.2 & 10.6 & \\
\hline LON & 11.4 & 13.7 & 14.2 & \\
\hline SE & 14.5 & 15.0 & 15.4 & \\
\hline SW & 12.2 & 9.2 & 9.5 & \\
\hline WM & 9.3 & 9.2 & 9.9 & \\
\hline NW & 12.7 & 13.7 & 12.6 & \\
\hline WAL & 6.1 & 8.8 & 5.4 & \\
\hline \multicolumn{5}{|l|}{ Education (\%) } \\
\hline Higher & 30.7 & 32.7 & 35 & \multirow[t]{5}{*}{ Labour Force Survey $2010^{b}$} \\
\hline A level & 11.1 & 14.8 & 21 & \\
\hline GCSE & 22.9 & 19.3 & 20 & \\
\hline Other & 15.3 & 16.1 & 12 & \\
\hline None & 20.0 & 17.0 & 11 & \\
\hline \multicolumn{5}{|l|}{ Employment (\%) } \\
\hline Full time & 66.5 & 72.9 & 66.8 & \multirow[t]{3}{*}{ Labour Force Survey 2011} \\
\hline Part time & 23.5 & 19.4 & 24.7 & \\
\hline Unemployed & 10.0 & 7.6 & 8.4 & \\
\hline \multicolumn{5}{|l|}{ Household size } \\
\hline One (\%) & 25.3 & 26.3 & 29.4 & \multirow[t]{6}{*}{ ONS 2011} \\
\hline Two (\%) & 33.1 & 35.3 & 35.0 & \\
\hline Three (\%) & 18.0 & 14.3 & 16.0 & \\
\hline Four (\%) & 14.0 & 12.9 & 13.2 & \\
\hline Five (\%) & 6.0 & 8.5 & 4.3 & \\
\hline $6+(\%)$ & 3.6 & 2.7 & 2.0 & \\
\hline
\end{tabular}

${ }^{\mathrm{a}}$ Column \% may not sum to $100 \%$ due to rounding.

${ }^{\mathrm{b}}$ National data only available to zero decimal point.

and that ethical approval was not required. Participants gave verbal consent to participate in the telephone survey. Data from the telephone survey was collated by the market research company and anonymised patient data were transferred to
PHE in a secure manner. Data were stored in a secured database in accordance with data protection rules and Caldicott principles. PHE has approval under Section 251 of the NHS Act 2006 to process confidential patient information for public 
Table 2. Self-reported risk factors (\%) in recruited participants under 65 years of age compared with national data (ImmForm), England and Wales, 2011-12

\begin{tabular}{lccc}
\hline Risk factor (\%) in under & $\begin{array}{c}\text { Two } \\
\text { surveys } \\
(\%) \\
(n=604)\end{array}$ & $\begin{array}{c}\text { Lost to } \\
\text { follow-up } \\
(\%) \\
(n=181)\end{array}$ & $\begin{array}{c}\text { National } \\
\text { ImmForm } \\
(16-64 \text { years } \\
\text { old })(\%)\end{array}$ \\
\hline Heart problem & 4.3 & 5.0 & 2.3 \\
\hline $\begin{array}{l}\text { Chest complaint/breathing } \\
\text { difficulties (including asthma) }\end{array}$ & 10.1 & 11.0 & 5.9 \\
\hline Kidney disease & 0.5 & 2.2 & 1.1 \\
\hline Liver disease & 0.7 & 0.0 & 0.6 \\
\hline Diabetes & 5.1 & 3.3 & 3.4 \\
\hline Stroke or a TIA & 1.8 & 0.0 & 0.6 \\
\hline Neurological condition & 1.7 & 0.5 & 0.7 \\
\hline Immunosuppression & 4.3 & 4.4 & 0.9 \\
\hline Any of the above & 22.7 & 22.1 & 13.2 \\
\hline
\end{tabular}

health purposes; separate ethical approval was not required for this study.

\section{Results}

The pre-peak survey was undertaken in the period from 13 to 19 October 2011. To obtain the desired survey size of 1000 individuals and their households during this period, a total of 63444 calls were made (to 32902 telephone numbers) recruiting a total of 1061 individuals, with 6247 refusing to participate and 64 abandoning the interview. The subsequent peak telephone survey took place between 9 and 25 March 2012. Eight hundred and thirty-four of the original 1061 (79\%) participants were successfully re-contacted and completed the follow-up survey, with an additional 50 refusing and one abandoning the interview.

The demographic characteristics of participants followed and lost to follow-up by geographical region, gender, age group, level of education, employment status and household size are outlined in Table 1. Overall the sample was broadly representative of the national population. By age in the group participating in both surveys, the 18-24 years old age group in particular was underrepresented and those over 65 years of age over-represented compared with national population estimates. By education, participants with no qualifications were over-represented and those completing secondary education to A level and higher education were under-represented. In terms of geographical region, the survey achieved a broadly representative sample from the different regions of the country with no one region being over- or underrepresented. Examining the group who were lost to follow-up after the first survey, 18-24 years old were more likely to be lost to follow-up (Table 1).

The self-reported risk status of individuals recruited in the survey compared with national data is shown in Table 2 . The results show an apparent over-representation of people with underlying chronic conditions under 65 years of age in the survey, with $22.7 \%$ of participants reporting an underlying clinical risk factor compared with $13.2 \%$ according to data from ImmForm. In particular, there were a larger number of people in the survey reporting chest complaints/breathing difficulties, including asthma compared with national data.
Influenza vaccination status of the study population found that overall, 315/1061 (29.7\%) reported having received an invitation from their GP to have influenza vaccine for the 2011/12 season - with $50 \%$ of those under 65 years $(68 / 137)$ in a self-defined clinical risk group recalling receiving an invite and $75 \%$ of those 65 years and above $(169 / 225)$. The proportion who reported that they were then vaccinated by age and risk group is outlined in Table 3. Ultimately, $55.8 \%$ of those under 65 years of age in a clinical risk group reported having received the 2011/12 seasonal influenza vaccine and $80.9 \%$ of 65 -year olds and above. The results from the telephone surveys quite closely mirror the ImmForm primary care-based vaccine uptake monitoring rates for the 2011/12 season, with the exception of pregnant women where vaccine uptake was estimated at $50.0 \%$ for the peak survey compared with $27.4 \%$ in ImmForm, although numbers were small. In terms of past vaccine history, there was some discordance between survey data for over 65-year olds and under 65 at risk compared with the national ImmForm data in both 2010/ 11 and 2009/10 with survey data generally lower than that in ImmForm (Table 3).

Self-reported respiratory illness rates are shown in Table 4. Seventy $(8.4 \%)$ of participants reported an ILI in the previous 2 weeks, and based on individual symptoms, 27 (3.2\%) had an ILI conforming to the modified WHO case definition. There were no large differences in the proportion self-reporting an ILI by age group except a slight excess in those 25-34 years of age. In addition, an excess in females was noted. The peak weekly ILI attack rates estimated through the telephone survey was $34.9 / 1000$ survey population in week 10 , the peak week and $12.0 / 1000$ survey population with the modified WHO definition.

The results of the peak survey show that the most common health-seeking behaviour in survey participants experiencing ILI was the use of non-prescription medicines, with $77.8 \%$ of those reporting a modified WHO-defined illness and $64.3 \%$ of those with a self-reported ILI reporting use of a nonprescription medicine (Table 4). The most common care-seeking behaviour after was to visit a GP surgery $(29.6 \%$ of those with modified WHO-defined illness and $22.9 \%$ of those with selfreported ILI).

Of the 1061 adults who were recruited to participate in the telephone survey, $574(54.1 \%)$ agreed to be re-contacted by PHE about providing biological samples to diagnose recent influenza infection.

\section{Discussion}

A population-based telephone survey pilot was successfully undertaken in England and Wales during the 2011/12 influenza season, a season dominated by circulation of influenza A $(\mathrm{H} 3 \mathrm{~N} 2)$, where the impact in terms of severity is usually found in older adults, in particular the elderly [25], demonstrating the feasibility of using this approach to rapidly assess new emerging pathogens, such as pandemic influenza. The demographic characteristics of the participants recruited and followed up using quota sampling were found to be broadly representative of the general population. The study design was able to successfully and rapidly recruit a cohort in 5 days and measure rates of acute respiratory illness in the general population, and provide a baseline of health care-seeking behaviour and calculate recent vaccine uptake. In addition, a large proportion of individuals indicated that they were prepared to provide biological samples to assist in the diagnosis of influenza. These components are essential to be able to 
Table 3. Self-reported influenza vaccination status (for 2011/12 vaccine, 2010/11 vaccine and 2009/10 vaccine) of telephone survey participants compared with national ImmForm data at pre-peak in week 41/42 and peak influenza activity, England and Wales, 2011-12

\begin{tabular}{|c|c|c|c|c|c|c|c|c|c|}
\hline \multirow{4}{*}{$\begin{array}{l}\text { Vaccine uptake } \\
\text { Group }\end{array}$} & \multicolumn{5}{|c|}{$2011 / 12(\%)$} & & & & \\
\hline & \multicolumn{3}{|c|}{ Pre-peak } & & & & & & \\
\hline & \multirow[b]{2}{*}{ Survey } & \multicolumn{2}{|c|}{ ImmForm } & \multicolumn{2}{|c|}{ Peak } & \multicolumn{2}{|c|}{$2010 / 11(\%)$} & \multicolumn{2}{|c|}{$2009 / 10(\%)$} \\
\hline & & week41 & week42 & Survey & ImmForm & Survey & ImmForm & Survey & ImmForm \\
\hline Under 65 (risk group) & 17.4 & 20.9 & 27.5 & 55.8 & 51.6 & 31.1 & 50.4 & 27.8 & 51.6 \\
\hline Over 65 & 48.6 & 38.1 & 48.7 & 80.9 & 74.0 & 80.1 & 72.8 & 53.1 & 72.4 \\
\hline Pregnant women & 22.2 & 8.0 & 11.0 & 50.0 & 27 & NA & NA & NA & NA \\
\hline
\end{tabular}

accurately estimate the severity of a new pathogen such as pandemic influenza.

Telephone surveys and quota sampling are being increasingly used as part of rapid population assessments [16, 17]. Although the limitations of valid inference from quota sampling are well recognised, there is an increasing recognition of their value when used appropriately [26]. Quota sampling uses prior knowledge of specific characteristics of the population to create strata to ensure representativeness of the sample. The target number of individuals to be obtained for each stratum is proportional to the corresponding stratum-specific population. There is also the potential for selection bias. In our study, however, the demographic characteristics of the quota sample were broadly representative of the general population for the various defined characteristics. In this instance, they can thus provide a seemingly representative sample with minimal missing data in the UK population.

We demonstrate that the telephone survey method was able to successfully and rapidly measure rates of acute ILI overall and by age and gender in the general population and also gather other key clinical and epidemiological data on these cases. The difference in self-reported ILI and ILI measured according to the WHO definition was noteworthy, with the more specific WHO case definition indicating a lower rate of illness, although there were no differences in the distribution by age group or gender for either case definition. Self-reported ILI rates in the general population through the telephone survey were considerably higher than those observed through sentinel GP reporting surveillance systems, where peak weekly ILI rate reported through Royal College of General Practitioners was 19.3/100000 in week 7 [25]. It is critical to capture such differences, particularly when integrated with laboratory confirmation, as it suggests that the true burden of respiratory illness due to influenza in the population is significantly underestimated, as was shown with the household-based FluWatch study [15] and to provide a more accurate estimate of the full spectrum of illness as part of measuring the true infection-severity ratio. In scenarios, where such cohorts are not established, the approach described in this paper provides an alternative option to rapidly recruit a cohort to enable such prospective studies to be done, particularly during future pandemics.

A significant proportion of symptomatic ILI cases meeting the modified WHO case definition in this study sought advice from their GP (30\%) or reported using non-prescription medication (78\%). These rates of consultation are not dissimilar to those observed in the FluWatch study undertaken in the period 2006-11 where $21 \%$ (95\% CI 17-25\%) of individuals with ILI reported going to consult their doctor [15]. Robust mechanisms to rapidly estimate health care-seeking behaviour will be an essential component of estimating the infection severity of future influenza pandemics and other emerging infections. The wide range of ages of those consulting their GP highlight that it is not possible to know whether these would have been recorded as ILI by their GP, though the FluWatch study suggests that the majority are not and that primary care underestimates the true burden of ILI in the community [15]. The specificity of defined ILI subjects that visit a GP requires more consideration in such surveys.

Self-reported vaccination uptake survey data generally compared well with population-based estimates of influenza vaccine uptake collected routinely through the primary care-based ImmForm web-based reporting system that is extracted in near real time from GP information systems [24]. During a pandemic, it is likely that delivery of a pandemic vaccine programme to a range of population groups will occur outside primary care, thus creating challenges to monitor population vaccine uptake. Random-digit-dialling telephone surveys have been successfully used in a variety of geographical settings to measure influenza vaccine uptake in different target groups, particularly in places where routine, real-time vaccine uptake monitoring is not established [27-29] or when vaccine is delivered outside of primary care. Such a design also provides the opportunity to investigate related issues such as risk factors for reduced vaccine uptake $[27,28]$.

A large proportion of participants agreed to be re-contacted by PHE about providing biological samples. Telephone surveys provide a potential platform to laboratory-confirmed influenza infection through established self-sampling tools [29]. In addition, they could be utilised to obtain population-based biological samples to measure immunity together with key clinical and epidemiological data such as vaccination status. Such nested laboratory surveys would be of critical value to measure seroincidence rates during a future pandemic, which are also required to estimate the infection severity. Future sampling studies need to be undertaken building on work such as this to determine the feasibility of collecting such biological samples, including approaches such as use of finger prick or oral fluid to measure influenza antibody levels. The latter investigations requires laboratory developmental work to create diagnostic tools with appropriate levels of sensitivity and specificity.

There were some limitations in this study. Quota-based sampling by definition is not a random sample of the population, and thus it is not possible to estimate the sampling error. Nonetheless, the sample did compare favourably to the general population for key criteria. There was an over-representation of 
Table 4. ILI attack rates (\%) by risk group, age group, gender, symptoms reported and healthcare-seeking behaviour for telephone survey participants, England and Wales, 2011-12

\begin{tabular}{|c|c|c|c|c|}
\hline & \multicolumn{2}{|c|}{$\begin{array}{c}\text { Self- } \\
\text { reported }\end{array}$} & \multicolumn{2}{|c|}{$\begin{array}{c}\text { Symptom } \\
\text { based }\end{array}$} \\
\hline & $N$ & $\%$ & $N$ & $\%$ \\
\hline \multicolumn{5}{|l|}{ ILI \% attack rate } \\
\hline Overall (all-age, $N=831$ ) & 70 & 8.4 & 27 & 3.2 \\
\hline \multicolumn{5}{|l|}{ Age group (years) } \\
\hline $18-24(N=63)$ & 6 & 9.5 & NA & \\
\hline $25-34(N=93)$ & 14 & 15.1 & NA & \\
\hline $35-44(N=147)$ & 12 & 8.2 & NA & \\
\hline $45-54(N=137)$ & 11 & 8.0 & NA & \\
\hline $55-64(N=161)$ & 13 & 8.1 & NA & \\
\hline $65-74(N=135)$ & 8 & 5.9 & NA & \\
\hline $75+(N=95)$ & 6 & 6.3 & NA & \\
\hline \multicolumn{5}{|l|}{$<65$ years at risk } \\
\hline All $(N=134)$ & 17 & 12.7 & NA & \\
\hline Vaccinated $(N=74)$ & 5 & 6.8 & NA & \\
\hline Unvaccinated $(N=60)$ & 12 & 20.0 & NA & \\
\hline \multicolumn{5}{|l|}{$65+$ year olds } \\
\hline All $(N=229)$ & 14 & 6.1 & NA & \\
\hline Vaccinated $(N=185)$ & 13 & 7.0 & NA & \\
\hline Unvaccinated $(N=44)$ & 1 & 2.3 & NA & \\
\hline \multicolumn{5}{|l|}{ Pregnant women $(n=6)$} \\
\hline All $(N=6)$ & 0 & 0.0 & NA & \\
\hline Vaccinated $(N=3)$ & 0 & 0.0 & NA & \\
\hline Unvaccinated $(N=3)$ & 0 & 0.0 & NA & \\
\hline \multicolumn{5}{|l|}{ Gender } \\
\hline Female $(N=460)$ & 45 & 9.8 & NA & \\
\hline Male $(N=371)$ & 25 & 6.7 & NA & \\
\hline & \multicolumn{2}{|c|}{$n=70$} & \multicolumn{2}{|c|}{$n=27$} \\
\hline \multicolumn{5}{|l|}{ Symptoms } \\
\hline Cough & 55 & 78.6 & 27 & 100.0 \\
\hline Sore throat & 49 & 70.0 & 25 & 92.6 \\
\hline Aching muscles & 48 & 68.6 & 22 & 81.5 \\
\hline Sudden fever & 31 & 44.3 & 27 & 100.0 \\
\hline Shortness of breath & 31 & 44.3 & 20 & 74.1 \\
\hline Other & 26 & 37.1 & 9 & 33.3 \\
\hline \multicolumn{5}{|l|}{ Behaviour } \\
\hline Use non-prescription medicines & 45 & 64.3 & 21 & 77.8 \\
\hline Visit GP surgery & 16 & 22.9 & 8 & 29.6 \\
\hline Speak to a pharmacist & 6 & 8.6 & 2 & 7.4 \\
\hline Look up symptoms on internet & 4 & 5.7 & 2 & 7.4 \\
\hline Visit $A$ and $E$ & 2 & 2.9 & 1 & 3.7 \\
\hline Visit walk-in/OOH clinic & 2 & 2.9 & 1 & 3.7 \\
\hline
\end{tabular}

Table 4. (Continued.)

\begin{tabular}{ccccccc}
\hline & \multicolumn{2}{c}{$\begin{array}{c}\text { Self- } \\
\text { reported }\end{array}$} & & \multicolumn{2}{c}{$\begin{array}{c}\text { Symptom } \\
\text { based }\end{array}$} \\
& $N$ & $\%$ & & $N$ & $\%$ \\
\hline Phone NHS Direct & 1 & 1.4 & 1 & 3.7 \\
\hline Use NHS Direct online & 1 & 1.4 & 1 & 3.7 \\
\hline Admitted to hospital & 0 & 0.0 & 0 & 0.0 \\
\hline
\end{tabular}

people $<65$ years with an underlying clinical risk factor. This overrepresentation may be attributable to the self-reported nature of the chronic conditions, particularly for underlying respiratory disease, the severity of which a GP may determine does not warrant vaccination. There may also be recall issues with self-reporting of historical vaccine uptake information from previous seasons. Some of the sub-groups, e.g. pregnant women were small in size, limiting the conclusions that can be made about results linked to these groups. Another key limitation of the study is that the recruitment of participants is restricted to those with a landline telephone number, although only a minority of households (about 12\%) do not have a landline and those with phones, who answer, may be distributed differently than the target population. These factors have the potential to introduce biases in the sample recruited, although the representativeness of this sample proved to be good for the demographic characteristics examined. If the proportion without a landline continues to increase, use of mobile phones as part of the sampling frame could potentially alleviate this issue.

Overall the telephone survey methodology provided a viable mechanism to rapidly recruit a cohort for longitudinal follow-up and to quickly gather key epidemiological data and potentially biological samples for seasonal influenza. It provides a potential tool to measure severity within the general population during future pandemics and for other emerging respiratory pathogens [30]. Further piloting on a larger scale is needed during seasonal influenza to test the scalability of this methodology for use in a pandemic and the feasibility of undertaking integrated biological sampling.

Acknowledgements. The authors would like to acknowledge and thank Ipsos MORI, the market research group, who were contracted by PHE to undertake the telephone survey presented in this paper. The design of the survey and the data capture tools, the data analysis and interpretation were undertaken by PHE. Nikolaos Panagiotopoulos provided helpful comments on an earlier draft. The authors would also like to thank all the members of the PHE Community Influenza Surveillance Steering Group.

Conflict of interest. None.

\section{References}

1. WHO Pandemic Influenza Severity Assessment (PISA). Available at http://www.who.int/influenza/surveillance_monitoring/pisa/pisaindicators/en/

2. Nicoll A et al. (2010) Experience and lessons from surveillance and studies of the 2009 pandemic in Europe. Public Health 124, 14-23.

3. HPA Epidemiological report of pandemic (H1N1) 2009 in the UK. Available at http://webarchive.nationalarchives.gov.uk/20140714113122/ http://www.hpa.org.uk/webc/HPAwebFile/HPAweb_C/1284475321350

4. Stoto MA (2012) The effectiveness of U.S. Public Health Surveillance Systems for situational awareness during the 2009 H1N1 pandemic: a retrospective analysis. PLOS ONE 7, e40984. 
5. Rutter P et al. (2014) Access to the NHS by telephone and Internet during an influenza pandemic: an observational study. BMJ Open 4, e004174.

6. Centers for Disease Control and Prevention (CDC) (2009) Outbreak of swine-origin influenza A (H1N1) virus infection - Mexico, March - April 2009. MMWR Morbidity Mortality Weekly Report 58(dispatch), 1-3.

7. Novel Swine-Origin Influenza A (H1N1) Virus Investigation Team (2009) Emergence of a novel swine-origin influenza A (H1N1) virus in humans. New England Journal of Medicine 360, 2605-2615.

8. Swerdlow DL, Finelli L and Bridges CB (2011) 2009 H1N1 influenza pandemic: field and epidemiologic investigations in the United States at the start of the first pandemic of the 21st century. Clinical Infectious Disease 52(Suppl 1), S1-S3.

9. Garske T et al. (2009) Assessing the severity of the novel influenza A/ H1N1 pandemic. British Medical Journal 339, b2840.

10. Laurie K et al. (2013) Influenza serological studies to inform public health actions: best practices to optimize timing, quality and reporting. Influenza and Other Respiratory Viruses 7, 211-224.

11. Department of Health (2010) Pandemic Influenza Preparedness Programme. Chief Medical Officer's Statistical Legacy Group.

12. Monto AS (1994) Studies of the community and family: acute respiratory illness and infection. Epidemiological Reviews 16, 351-373.

13. Adler AJ et al. (2014) Incidence and risk factors for influenza-like-illness in the UK: online surveillance using Flusurvey. BMC Infectious Diseases 14, 232.

14. Hardelid P et al. (2010) Assessment of baseline age-specific antibody prevalence and incidence of infection to novel influenza A/H1N1 2009. Health Technology Assessment 14, 115-192.

15. Hayward AC et al. (2014) Comparative community burden and severity of seasonal and pandemic influenza: results of the Flu Watch cohort study. Lancet Respiratory Medicine 2, 445-454.

16. Payne L et al. (2005) 'Did you have flu last week?' A telephone survey to estimate a point prevalence of influenza in the Swedish population. Euro Surveillance 10, 241-244.

17. Malone JL, Madjid M and Casscells SW (2008) Telephone survey to assess influenza-like illness, United States, 2006. Emerging Infectious Diseases 14, 129-135.

18. Rubin J et al. (2009) Public perceptions, anxiety, and behaviour change in relation to the swine flu outbreak: cross sectional telephone survey. British Medical Journal 339, b2651.
19. Sedgewick P (2012) Proportional quota sampling. British Medical Journal 345, e6336.

20. Percentage of households with a landline in the UK from 1970 to 2016. Available at https://www.statista.com/statistics/289158/telephonepresence-in-households-in-the-uk/

21. World Health Organisation. Influenza (Seasonal) Fact Sheet No. 211. Available at http://www.who.int/mediacentre/factsheets/fs211/en/ (Accessed 7 March 2012).

22. Annual Mid-Year Population Estimates 2001 to 2010. Available at https://www.ons.gov.uk/peoplepopulationandcommunity/populationand migration/populationestimates/bulletins/annualmidyearpopulationestimates/ 2013-12-17

23. Labour Force Annual Survey 2011. Available at https://discover.ukdata service.ac.uk/catalogue/?sn $=7291$

24. Gates P et al. (2009) Collection of routine national seasonal influenza vaccine coverage data from GP practices in England using a web-based collection system. Vaccine 27, 6669-6677.

25. Surveillance of influenza and other respiratory pathogens in the UK 2011/12. Available at http://webarchive.n-ationalarchives.gov.uk/2014 0714113118/http://www.hpa.org.uk/webc/HPAwebFile/HPAweb_C/13171 34705939

26. Vallance JK et al. (2014) Utility of telephone survey methods in population-based health studies of older adults: an example from the Alberta Older Adult Health Behaviour (ALERT) study. BMC Public Health 14, 486.

27. Böhmer MM et al. (2012) Barriers to pandemic influenza vaccination and uptake of seasonal influenza vaccine in the post-pandemic season in Germany. BMC Public Health 12, 938.

28. Vaux S et al. (2011) Influenza vaccination coverage against seasonal and pandemic influenza and their determinants in France: a cross-sectional survey. BMC Public Health 11, 30.

29. Müller D, Nguyen-Van-Tam JS and Szucs TD (2006) Influenza vaccination coverage rates in the UK: a comparison of two monitoring methods during the 2002-2003 and 2003-2004 seasons. Public Health 120, 10741080.

30. Van Kerkhove MD et al. HO informal network for mathematical modelling for pandemic influenza H1N1 2009 (Working Group on Data Needs) (2010) Studies needed to address public health challenges of the 2009 H1N1 influenza pandemic: insights from modeling. PLoS Medicine 7, e1000275. 